\title{
Privação de liberdade ou direitos: revisão integrativa sobre tuberculose no sistema penitenciário brasileiro
}

\author{
Jaqueline de Almeida Moreira ${ }^{1}$ \\ Mario Ribeiro Alves ${ }^{2}$ \\ Universidade Federal de Mato Grosso
}

Resumo: O presente artigo visa compreender a atenção à saúde da População Privada de Liberdade com tuberculose no Sistema Penitenciário Brasileiro, via revisão bibliográfica integrativa. Foram analisados 155 artigos e o levantamento dos estudos foi realizado em janeiro e fevereiro de 2020, buscando artigos de 2010 a 2019. A seleção dos artigos ocorreu por critérios de inclusão e exclusão, baseada em três etapas. Foram descritas como obstáculos à saúde da PPL as condições estruturais precárias das celas, as más condições sanitárias, superlotação, limitação à luz solar, ventilação inadequada como obstáculo à saúde da PPL. Observou-se serem necessárias capacitações aos profissionais de saúde para alinhamento aos programas e planos implementados para a saúde da PPL. Metade dos estudos sinaliza que a maioria da população provém de um contexto em que as iniquidades em saúde se fazem presentes, mostrando necessidade de ações sobre essas iniquidades.

Palavras-chave: população privada de liberdade; tuberculose; determinantes sociais de saúde; iniquidades; sistema penitenciário.

\footnotetext{
${ }^{1}$ Possui Graduação em Saúde Coletiva pela Universidade Federal de Mato Grosso, com experiência em temas relacionados à saúde da população privada de liberdade.

${ }^{2}$ Geógrafo e Doutor em Epidemiologia em Saúde Pública pela Fundação Oswaldo Cruz. Atualmente realiza pós-doutorado em Saúde Coletiva pela Universidade Federal de Mato Grosso.
} 


\title{
Deprivation of freedom or rights: integrative review of tuberculosis in the Brazilian penitentiary system
}

\begin{abstract}
This article aims to understand the health care of Liberty Deprived Population with tuberculosis in the Brazilian Penitentiary System, via an integrative bibliographic review. The articles were analyzed, and the survey of the studies was carried out in January and February 2020, looking for articles from 2010 to 2019. Articles were selected according to inclusion and exclusion criteria, based on three stages. The authors described the precarious structural conditions of the cells, poor sanitary conditions, overcrowding, limited sunlight, inadequate ventilation as an obstacle to the health of the LDP. It was observed that training for health professionals is necessary to align with the programs and plans implemented for PPL health. Half of the studies indicated that most of the population comes from a context in which health inequities are present daily, showing the need for actions on these inequities.
\end{abstract}

Keywords: liberty deprived population; tuberculosis; social determinants of health; inequities; penitentiary system.

\section{Privación de libertad o derechos: revisión integradora de la tuberculosis en el sistema penitenciario brasileño}

\begin{abstract}
Resumen: Este artículo tiene como objetivo comprender la atención de salud de la Población Privada de Libertad con tuberculosis en el Sistema Penitenciario Brasileño, a través de una revisión bibliográfica integradora. Se analizaron 155 artículos y la encuesta de los estudios se realizó en enero y febrero de 2020, buscando artículos de 2010 a 2019. Los artículos fueron seleccionados según criterios de inclusión y exclusión, con base en tres etapas. Han sido descritas las precarias condiciones estructurales de las celdas, malas condiciones sanitarias, hacinamiento, escasez de luz solar, ventilación inadecuada como un obstáculo para la salud de las PPL. Se observó que la capacitación de los profesionales de la salud es necesaria para alinearse con los planes implementados para la salud de PPL. Mitad de los estudios indican que la mayoría de la población proviene de un contexto en el que las inequidades en salud se presentan, mostrando necesidad de acciones sobre estas inequidades.
\end{abstract}

Palabras clave: población privada de libertad; tuberculosis; los determinantes sociales de la salud; inequidades; sistema penitenciario. 
$\mathrm{A}$ assistência à saúde da população privada de liberdade (PPL) foi instituída como direito por meio da Lei de Execuções Penais (LEP) 7.210, de 11 de julho de 1984, sendo de caráter preventivo e curativo e devendo abarcar atendimento médico, farmacêutico e odontológico, necessitando de autorização da direção da unidade penitenciária em casos de necessidade de atendimento diferente do oferecido pelo estabelecimento penal ou em sua ausência (BRASIL, 1984).

O que assegura o direito ao acesso da PPL às ações e aos serviços de saúde está disposto em legislações anteriores como: a Lei 7.210/1984, que institui a LEP, aqui já citada; a Constituição Federal de 1988; a Lei 8.080 de 1990, que regulamenta o Sistema Único de Saúde (SUS); e a Lei 8.142 de 1990, que dispõe sobre a participação da comunidade na gestão do SUS (BRASIL, 2004).

Ao analisar o nascimento das prisões e as formas de punição, Foucault (1977) recorda o século XVII, quando as penas físicas eram aplicadas sob formato de grandes espetáculos, como um cerimonial aberto ao público, exposto à luz do dia. No entanto, já no século XVIII, a punição deixou de ser uma cena e sua perspectiva espetacular passou a ser algo negativo e reprovável. A mudança na exposição do suplício veio acompanhada do afrouxamento da severidade penal e de um deslocamento da ação punitiva, algo que se conforma de modo mais sólido em meados do século XIX. Esse deslocamento da ação punitiva colocava como alvo não mais a punição do corpo, mas a punição da alma dos sujeitos infratores.

A questão da disciplina, do poder e da punição social nortearam reflexões teóricas e filosóficas de modo bastante incisivo durante boa parte do século XX (FOUCAULT, 1977; 1979; GOFFMAN, 1961) e o Brasil não ficou de fora desse amplo espaço de debates. Com relação ao Sistema Penitenciário Brasileiro, Adorno (1991) observa que os desafios encontrados na década de 1980, com o crescimento da criminalidade urbana e o esgotamento do poder judiciário (que foi se tornando cada vez mais seletivo), foram conduzindo à revisão e ao abrandamento de penas em crimes considerados menos graves, na contrapartida de uma perceptível rigidez no julgamento de crimes graves e de casos de reincidência, com o intuito de conter a superpopulação encarcerada.

Porém, a aplicabilidade dessas medidas não foi suficiente para aliviar o já sobrecarregado sistema penitenciário, que continuou apresentando um quadro de má qualidade de vida; superlotação; condições sanitárias rudimentares; alimentação deteriorada; precária assistência médica, judiciária, educacional e profissional (ADORNO, 1991: 70-71). A precarização da vida da pessoa encarcerada foi se tornando, então, objeto de reflexão em todo o território nacional e a questão da saúde tornou-se um ponto de especial atenção (ADORNO, 1991).

A Organização Mundial da Saúde (OMS) apresenta que a maior parte das doenças e as iniquidades em saúde acontecem devido aos determinantes sociais de saúde (DSS) que agregam as condições em que as pessoas nascem, vivem, crescem, trabalham e envelhecem (OMS, 2011). 
Desde 2003, o Brasil figura como um dos países com o maior número de casos de tuberculose no mundo e, embora seu diagnóstico e tratamento sejam realizados gratuitamente e de forma universal pelo SUS, ainda se registra uma média de 4.500 óbitos e 69 mil novos casos por ano (BRASIL, 2017).

Este trabalho toma por base o conceito de saúde pacificado na $8^{\text {a }}$ Conferência Nacional de Saúde, que entende a saúde como resultante das condições de alimentação, habitação, educação, renda, meio ambiente, trabalho, lazer, acesso a serviços de saúde, entre outros. O direito à saúde significa a garantia, pelo Estado, de condições dignas de vida e acesso universal e igualitário às ações e serviços de promoção, proteção e recuperação de saúde, em todos os níveis e a todos os habitantes, levando ao pleno desenvolvimento do ser humano em sua individualidade (BRASIL, 1986).

A motivação para a realização deste estudo vem da tentativa de compreender como são tratados pelo Estado os direitos da população privada de liberdade, principalmente o direito à saúde. Por considerar de extrema relevância a relação entre a tuberculose e as noções de vulnerabilidade em saúde e risco epidemiológico, pretende-se conhecer as condições de saúde da população privada de liberdade em relação à tuberculose e os determinantes de adoecimento, por meio de revisão bibliográfica integrativa, a fim de se verificar a presença do Estado como um tutor desses sujeitos, durante a execução da pena de privação de liberdade, resguardando a dignidade da pessoa humana em seu acesso à saúde.

Este trabalho tem como objetivo compreender a atenção à saúde da PPL com tuberculose no Sistema Penitenciário Brasileiro, por meio de uma revisão bibliográfica integrativa, identificando evidências sobre condições de saúde e determinantes de adoecimento relacionados à tuberculose da PPL.

\section{Métodos}

Foi realizada revisão integrativa sobre saúde de PPL, com tuberculose, no sistema penitenciário brasileiro, buscando artigos dos anos de 2010 a 2019, nas bases de dados SciELO e LILACS, esta última através da Biblioteca Virtual em Saúde (BVS), utilizando os descritores: tuberculose, sistema penitenciário, sistema prisional.

Foram analisados 155 artigos e o levantamento dos estudos foi realizado em janeiro e fevereiro de 2020. Como critérios de inclusão foram selecionados os artigos: nos idiomas português ou inglês; com coleta de dados no Brasil; com o termo saúde no sistema penitenciário/prisional e tuberculose; e com publicação nos últimos dez anos. Os critérios de exclusão foram: não apresentar temática referente à saúde dos presos e tuberculose e artigos com mais de dez anos. Os artigos duplicados nas bases de dados consultadas foram delimitados para não alterar o quantitativo de publicações encontradas.

A seleção dos artigos ocorreu por critérios de inclusão e exclusão, baseada em três etapas. Na primeira etapa foram considerados idioma e ano de publicação. Na segunda, as publicações foram selecionadas mediante leitura do título e resumo para inclusão das publicações que correspondem ao tema de estudo proposto. Na terceira etapa, os artigos foram lidos na íntegra e avaliados critérios de inclusão e exclusão de acordo com a relação existente com o tema a ser estudado.

Por utilizar dados públicos secundários que não envolvem seres humanos e que não identificam estabelecimentos ou instâncias, este estudo não se enquadra nos preceitos da Resolução 466/2012 do Conselho Nacional de Saúde (BRASIL, 2012), não sendo necessário ter sido submetido ao Comitê de Ética. 


\section{Resultados}

Durante a seleção foram analisadas 155 publicações, destes, 19 foram selecionados para leitura na íntegra. Após esta etapa, 13 artigos foram excluídos por não abordarem a tuberculose no sistema penitenciário. Assim, a seleção final foi composta por 6 artigos, conforme Quadro 1.

Quadro 1: Número de publicações selecionadas através de etapas de seleção, utilizando as palavras-chave "sistema penitenciário" e "tuberculose" ou "sistema prisional".

\begin{tabular}{|c|c|c|c|c|}
\hline BASE DE DADOS & ENCONTRADOS & ETAPA 1 & ETAPA 2 & ETAPA 3 \\
\hline SciELO & 180 & 76 & 14 & 6 \\
\hline LILACS & 97 & 79 & 5 & 0 \\
\hline Total & 277 & 155 & 19 & 6 \\
\hline
\end{tabular}

Os Quadros 2 e 3 caracterizam os artigos e as pesquisas analisados, estando organizados por autor, tipo de produção, objetivo, metodologia e resultado (Quadro 2) e autor, cenário de estudo e população estudada (Quadro 3).

Quadro 2: Caracterização das pesquisas sobre tuberculose no sistema penitenciário brasileiro, de 2010 a 2019, segundo autor e ano de publicação, tipo de produção científica, objetivo, metodologia e resultados.

\begin{tabular}{|c|c|c|c|c|}
\hline AUTOR/ANO & $\begin{array}{l}\text { TIPO DE PRODU- } \\
\text { ÇÃO CIENTÍFICA }\end{array}$ & OBJETIVO & METODOLOGIA & RESULTADO \\
\hline $\begin{array}{l}\text { CARDINS et al., } \\
2019\end{array}$ & $\begin{array}{l}\text { Artigo (Escola Anna } \\
\text { Nery) }\end{array}$ & $\begin{array}{l}\text { Avaliar o acesso e o } \\
\text { uso racional de medi- } \\
\text { camentos essenciais e } \\
\text { estratégicos da PPL }\end{array}$ & $\begin{array}{l}\text { Estudo qualitativo, re- } \\
\text { alizado entre os me- } \\
\text { ses de fevereiro e } \\
\text { agosto de } 2016\end{array}$ & $\begin{array}{l}\text { Por mais que as políticas } \\
\text { garantam o acesso e uso } \\
\text { racional de medicamentos } \\
\text { nas penitenciárias, exis- } \\
\text { tem casos de falta de me- } \\
\text { dicamentos essenciais e } \\
\text { estratégicos }\end{array}$ \\
\hline $\begin{array}{l}\text { CORDEIRO et al., } \\
2018\end{array}$ & $\begin{array}{l}\text { Artigo (Avances en } \\
\text { Enfermería) }\end{array}$ & $\begin{array}{l}\text { Analisar as principais } \\
\text { patologias que acome- } \\
\text { tem os detentos de um } \\
\text { Complexo Prisional de } \\
\text { Recife, refletindo à luz } \\
\text { da literatura sobre as } \\
\text { concepções de enfer- } \\
\text { magem que permeiam } \\
\text { tais agravos }\end{array}$ & $\begin{array}{l}\text { Estudo documental, } \\
\text { de natureza explorató- } \\
\text { ria e descritiva, com } \\
\text { abordagem qualitativa }\end{array}$ & $\begin{array}{l}\text { Os dados obtidos, em re- } \\
\text { lação às doenças notifica- } \\
\text { das nos detentos estão } \\
\text { em consonância com os } \\
\text { evidenciados pelo Levan- } \\
\text { tamento Nacional de Infor- } \\
\text { mações Penitenciárias, no } \\
\text { qual em primeiro se tem } \\
\text { HIV-Aids, em seguida sífi- } \\
\text { lis, hepatites e tuberculose }\end{array}$ \\
\hline $\begin{array}{l}\text { BARBOSA et al., } \\
2014\end{array}$ & $\begin{array}{l}\text { Artigo (Escola Anna } \\
\text { Nery) }\end{array}$ & $\begin{array}{l}\text { Descrever aspectos } \\
\text { estruturais e caracte- } \\
\text { rísticas do trabalho na } \\
\text { atenção básica à sa- } \\
\text { úde em penitenciárias } \\
\text { masculinas do estado } \\
\text { da Paraíba e fornecer } \\
\text { subsídios para a atua- } \\
\text { ção do enfermeiro } \\
\text { nesse cenário }\end{array}$ & $\begin{array}{l}\text { Estudo descritivo } \\
\text { transversal, com abor- } \\
\text { dagem quantitativa }\end{array}$ & $\begin{array}{l}\text { Verificou-se que o atendi- } \\
\text { mento à demanda espon- } \\
\text { tânea é realizado em } \\
100 \% \text { das unidades de sa- } \\
\text { úde. Quanto às ações de } \\
\text { atenção básica em saúde } \\
\text { desenvolvidas no âmbito } \\
\text { do Sistema Penitenciário } \\
\text { do estado da Paraíba, } \\
\text { destaca-se o diagnóstico } \\
\text { e tratamento de diabetes, } \\
\text { hanseníase, tuberculose e } \\
\text { hipertensão, sendo indi- } \\
\text { cado em pelo menos cinco } \\
\text { (83\%) dos seis estabeleci- } \\
\text { mentos }\end{array}$ \\
\hline
\end{tabular}




\begin{tabular}{|c|c|c|c|c|}
\hline $\begin{array}{l}\text { NAVARRO et al., } \\
2016\end{array}$ & $\begin{array}{l}\text { Artigo (Jornal Brasi- } \\
\text { leiro de Pneumolo- } \\
\text { gia) }\end{array}$ & $\begin{array}{l}\text { Estimar a prevalência } \\
\text { e os fatores associa- } \\
\text { dos com a infecção la- } \\
\text { tente por Mycobacte- } \\
\text { rium tuberculosis em } \\
\text { PPL no principal polo } \\
\text { prisional de Minas Ge- } \\
\text { rais }\end{array}$ & $\begin{array}{l}\text { Estudo de corte trans- } \\
\text { versal }\end{array}$ & $\begin{array}{l}\text { A prevalência de infecção } \\
\text { latente por Mycobacterium } \\
\text { tuberculosis foi alta entre } \\
\text { PPL das duas maiores } \\
\text { unidades prisionais / peni- } \\
\text { tenciárias de Minas Gerais } \\
\text { e que estava associada ao } \\
\text { relato de contato com pa- } \\
\text { cientes com tuberculose } \\
\text { dentro dessas unidades e } \\
\text { ao uso de drogas inaláveis }\end{array}$ \\
\hline $\begin{array}{l}\text { OLIVEIRA et al., } \\
\text { 2015a }\end{array}$ & $\begin{array}{l}\text { Artigo (Cadernos de } \\
\text { Saúde Pública) }\end{array}$ & $\begin{array}{l}\text { Analisar a implantação } \\
\text { do programa de con- } \\
\text { trole da tuberculose no } \\
\text { sistema penitenciário } \\
\text { de dois estados brasi- } \\
\text { leiros }\end{array}$ & $\begin{array}{l}\text { Estudo de casos múlti- } \\
\text { plos foi desenvolvido } \\
\text { no período de dezem- } \\
\text { bro de } 2010 \text { a junho de } \\
2012 \text {, com abordagem } \\
\text { qualitativa, para ope- } \\
\text { racionalizar o modelo } \\
\text { lógico e modelo teó- } \\
\text { rico do programa, } \\
\text { apresentados no es- } \\
\text { tudo de avaliabilidade }\end{array}$ & $\begin{array}{l}\text { Mostrou que as ações do } \\
\text { programa de controle da } \\
\text { tuberculose não estão to- } \\
\text { talmente implantadas nas } \\
\text { prisões estudadas em } \\
\text { dois dos estados com } \\
\text { maior população prisional, } \\
\text { altas taxas de incidência } \\
\text { de tuberculose e situação } \\
\text { econômica melhor do que } \\
\text { a média do país }\end{array}$ \\
\hline $\begin{array}{l}\text { OLIVEIRA et al., } \\
\text { 2015b }\end{array}$ & $\begin{array}{l}\text { Artigo (Revista de } \\
\text { Saúde Pública) }\end{array}$ & $\begin{array}{l}\text { Analisar a influência } \\
\text { das características } \\
\text { contextuais do controle } \\
\text { da tuberculose nas pri- } \\
\text { sões e a influência dos } \\
\text { graus de implantação } \\
\text { do programa nos efei- } \\
\text { tos observados }\end{array}$ & $\begin{array}{l}\text { Estudo de múltiplos } \\
\text { casos com abordagem } \\
\text { qualitativa }\end{array}$ & $\begin{array}{l}\text { Os problemas detectados } \\
\text { no programa de controle } \\
\text { de tuberculose prisional } \\
\text { não impedem de exercer } \\
\text { seu papel na redução da } \\
\text { morbimortalidade por tu- } \\
\text { berculose e diminuir as si- } \\
\text { tuações de risco no sis- } \\
\text { tema prisional }\end{array}$ \\
\hline
\end{tabular}

Quadro 3: Caracterização das pesquisas que melhor atenderam às necessidades do estudo, segundo autor, cenário de estudo e população estudada.

\begin{tabular}{|c|c|c|}
\hline AUTOR & CENÁRIO DE ESTUDO & POPULAÇÃO DE ESTUDO \\
\hline CARDINS et al., 2019 & $\begin{array}{l}\text { Sete penitenciárias do estado da Para- } \\
\text { iba }\end{array}$ & $\begin{array}{l}\text { Um representante da Coordenação de } \\
\text { Saúde Penitenciária da Paraíba, seis } \\
\text { médicos, sete enfermeiros e quarenta e } \\
\text { três PPL em uso de medicamentos es- } \\
\text { sências e/ou estratégicos }\end{array}$ \\
\hline CORDEIRO et al., 2018 & $\begin{array}{l}\text { Complexo Prisional Estadual, localizado } \\
\text { em Recife, Pernambuco }\end{array}$ & $\begin{array}{l}\text { PPL atendidas no ambulatório do com- } \\
\text { plexo prisional, durante o ano de } 2015 \text {. } \\
\text { A amostra foi composta de } 113 \text { prontuá- } \\
\text { rios }\end{array}$ \\
\hline BARBOSA et al., 2014 & $\begin{array}{l}\text { Seis estabelecimentos de assistência à } \\
\text { saúde, integrados a penitenciárias mas- } \\
\text { culinas subordinadas à Secretaria de } \\
\text { Estado da Administração Penitenciária } \\
\text { (SEAP) }\end{array}$ & $\begin{array}{l}\text { Diretores institucionais das penitenciá- } \\
\text { rias localizadas nas cidades de Cam- } \\
\text { pina Grande (2), Guarabira (1), João } \\
\text { Pessoa (2) e Santa Rita }\end{array}$ \\
\hline NAVARRO et al., 2016 & $\begin{array}{l}\text { Duas maiores unidades prisionais / pe- } \\
\text { nitenciárias, localizadas no município de } \\
\text { Ribeirão das Neves. Minas Gerais }\end{array}$ & $\begin{array}{l}1.492 \text { detentos, sendo que } 1.120 \text { foram } \\
\text { incluídos na amostra final }\end{array}$ \\
\hline OLIVEIRA et al., 2015a & $\begin{array}{l}\text { Sistema de saúde penitenciária de dois } \\
\text { estados brasileiros com grande popula- } \\
\text { ção carcerária }\end{array}$ & $\begin{array}{l}\text { Duas unidades prisionais para cada } \\
\text { caso e um hospital penal em um dos ca- } \\
\text { sos }\end{array}$ \\
\hline OLIVEIRA et al., 2015b & $\begin{array}{l}\text { Sistema de saúde penitenciária de dois } \\
\text { estados }\end{array}$ & $\begin{array}{l}\text { Duas unidades prisionais de cada es- } \\
\text { tado. Em um dos casos, foi incluído um } \\
\text { hospital penal entre as unidades de aná- } \\
\text { lise }\end{array}$ \\
\hline
\end{tabular}


à luz solar, ventilação inadequada, gerando condições favoráveis para proliferação de doenças infectocontagiosas.

Embora entre sadia na unidade penitenciária, a PPL não sai sem alguma doença ou com sua saúde/resistência física fragilizada, em decorrência de fatores estruturais, como as já citadas, superlotação de celas, precariedade e insalubridade delas e ainda má alimentação, uso de drogas, sedentarismo e falta de higiene. Além do mais, ao passar para a tutela do Estado, o preso perde não só o seu direito à liberdade, como também outros direitos não alcançados pela sentença (ASSIS, 2007).

A implantação das medidas de controle da tuberculose nas unidades penitenciárias, da maneira que estão postas pelo Plano Nacional de Saúde do Sistema Penitenciário (PNSSP) e Programa Nacional de Controle da Tuberculose (PNCT), ocorrem em um ambiente adverso, onde os direitos assegurados pela LEP não estão de fato garantidos (OLIVEIRA et al., 2015b).

Cordeiro et al. (2018) nos recordam que o PNSSP indica a necessidade da busca ativa, bem como o Tratamento Diretamente Observado (TODO), diagnóstico precoce e a notificação compulsória, além da investigação dos comunicantes para tuberculose e hanseníase. Cardins et al. (2019), demonstraram que, em relação à medicação para o tratamento da tuberculose, os profissionais da saúde relataram falta de tempo para realizar o TDO.

Dos dois casos analisados por Navarro et al. (2016), um realizava o TDO por profissionais da saúde nos dias de semana (ficando nos finais de semana sob a responsabilidade do agente de segurança) e, no outro, o profissional de saúde fazia a entrega dos medicamentos semanalmente, com tratamento autoadministrado (sob o argumento de parecer coercitivo e autoritário realizar o TDO com uma população já encarcerada).

Por estes achados, percebe-se que além da insalubridade e das más condições das celas, a PPL acaba tendo que contar com a percepção da equipe de saúde ou dos agentes de segurança para um tratamento indicado e eficaz. A não realização do TDO pode gerar prejuízos ao tratamento, uma vez que não há como ter a garantia de que a PPL segue realizando o seu tratamento corretamente, colocando a pessoa que está sendo tratada em risco, assim como os demais.

Para os agentes de segurança, as pessoas privadas de liberdade, muitas vezes, passam imagem de interlocutores não confiáveis, o que acarreta uma desvalorização das queixas destes quanto a seu estado de saúde, sendo assim necessária a apresentação de sintomas verificáveis (DIUANA, 2008).

Os agentes de segurança se mostram hostis quanto aos direitos dos presos, assim como a maior parte da população. Porém, agentes que receberam treinamento sobre tuberculose (ou os que eram da área da saúde) percebiam a importância das ações para o indivíduo e para o coletivo, demonstrando necessidade desse tipo de ação para que a tuberculose se torne uma preocupação compartilhada (OLIVEIRA et al., 2015b).

Nas penitenciárias, fatores como insalubridade, prevalência de agravos, dificuldade de acesso e uso indevido de medicamentos, comprometem a assistência adequada, apesar da política nacional de assistência farmacêutica ter o dever de garantir o fornecimento de medicamentos que atendam a adequada prescrição. A superlotação dos presídios está entre os prováveis motivos da escassez de medicamentos (CARDINS et al., 2019).

O sistema prisional no Brasil chegou a um ponto precário, não havendo unidade prisional que esteja sob responsabilidade do Estado que tenha número igual ou inferior de presos ao de vagas. Assim, todas as unidades estão superlotadas, 
com os maiores índices de reincidência do mundo (ANDRADE; FERREIRA, 2014).

Além do mais, os poucos instrumentos e insumos fazem com que o sistema penitenciário tenha uma estrutura precária, não propiciando condições favoráveis para a promoção da saúde, o que também dificulta esse acesso é o fato de a demanda ser incompatível com a quantidade e a qualificação dos profissionais, o que acaba por comprometer a universalização e a integralização das ações na atenção primária (CARDINS et al., 2019). Por isso, faz-se necessário um adequado suporte material de equipamentos, instrumentos, materiais, insumos e medicamentos para que as ações de saúde sejam implementadas (BARBOSA et al., 2014).

A segurança nas unidades penitenciárias pode influenciar os aspectos administrativos, como no processo de cadastramento das PPL junto aos estabelecimentos de saúde e isso pode repercutir nas lacunas que ainda existem nos serviços de saúde implantados no sistema penitenciário (BARBOSA et al., 2014).

O trabalho de Cordeiro et al. (2018) demonstrou que 49,56\% da PPL não possuíam doenças prévias cadastradas, gerando subnotificação, que é um problema que dificulta a análise da situação de saúde e acaba por prejudicar o planejamento das ações para controle de doenças. Foi observado, ainda, que 77,88\% da PPL não teve nenhuma patologia notificada, o que ratifica o problema de subnotificação e falta de registro nos prontuários.

É preciso adequar o cadastramento da PPL junto aos estabelecimentos de saúde, para que se possam desenvolver ações de promoção da saúde e assistência adequada aos problemas mais frequentes dessa população bem como atingir o conhecimento, a prevenção, o controle e o tratamento de doenças (BARBOSA et al., 2014).

Cabe aqui lembrar que a tuberculose está incluída na relação de agravos de notificação compulsória, porém sem o devido cadastro da PPL junto aos estabelecimentos de saúde, não há como fazer o controle e o planejamento das ações junto a essa população. Além disso, a dispensa de remédios para o tratamento depende deste cadastramento.

Planejamento, gerência e financiamento contínuo de ações de saúde são elementos essenciais para o controle da endemia no contexto prisional; porém, são escassos os recursos destinados às necessidades sanitárias e há pouca participação dos gerentes e profissionais de saúde nas decisões sobre a aplicação desses recursos, junto com o aumento continuado do quantitativo de presos. Todos esses fatores contribuem para a desmotivação de muitos dos profissionais que ali estão (OLIVEIRA et al., 2015b).

A quantidade insuficiente de enfermeiros traz prejuízo à atuação dos profissionais da saúde no sistema penitenciário (CORDEIRO et al., 2018). Devemos destacar, também, o número de profissionais de saúde desmotivados com as condições de trabalho e sem treinamento periódico, investimento insuficiente e a carência de transporte e escolta adequados às ações de saúde extramuros (OLIVEIRA et al., 2015a).

Para Barbosa et al. (2014), compõe as dificuldades da prática dos enfermeiros as questões estruturais e organizacionais das unidades de saúde. Cabe ainda dizer que a ausência de capacitação da equipe de saúde nas ações de controle da tuberculose e a dificuldade de acesso aos serviços de saúde, também contribuem para inviabilizar a realização de uma ação eficiente (NAVARRO et al., 2016). 
$\mathrm{O}$ isolamento do paciente em privação de liberdade deve ocorrer quando o caso é identificado no ingresso deste à unidade penitenciária, em casos de resistência e quando não há condições de tratamento pela unidade, porém, este isolamento não é indicado quando o caso é identificado na população já encarcerada, pois as pessoas em contato com aquele paciente já foram expostas ao risco de infecção, o isolamento tende a desencorajar a busca por diagnóstico de outras pessoas presas em decorrência da limitação de circulação e pelo fato de que logo nas primeiras semanas o risco de contágio diminui (BRASIL, 2018).

Para Sánchez et al. (2006), são muitos os obstáculos para o controle da tuberculose nas prisões, entre eles a subvalorização dos sintomas, falta de recursos, dificuldade de acesso e, principalmente, a restrição de autonomia dos presos nas ações de prevenção e no seu tratamento. Diz, ainda, que enquanto esses indivíduos presos não se tornarem os atores centrais de sua saúde, a tuberculose segue sendo uma fatalidade, e que a promoção da saúde nesta luta deve ser conjunta a todas as pessoas expostas à tuberculose, como outros presos, familiares, agentes de segurança, profissionais da saúde e outros.

Se o perfil demográfico da população prisional brasileira é mesmo um reflexo da marginalização histórica da relação entre o cidadão e o Estado, como observam Soares Filho e Bueno (2016), no artigo "Demografia, vulnerabilidade e direito à saúde da população prisional brasileira", a questão da "vulnerabilidade" se coloca como um ponto central do desequilíbrio dessa equação. A já tão citada precariedade arquitetônica das estruturas penitenciárias, juntamente com a superlotação carcerária e a precariedade em termos de higiene e alimentação contribuem sobremaneira com a proliferação de epidemias e o desenvolvimento de patologias e psicopatologias no ambiente prisional brasileiro.

O quadro é piorado quando ao alto índice de iniquidades e vulnerabilidades em saúde se agregam as faltas de procedimentos adequados no diagnóstico da tuberculose e, posteriormente, no seu tratamento no contexto prisional. Os artigos analisados na revisão integrativa pretendida no presente estudo são categóricos ao afirmarem que alguns dos fatores que podem contribuir para a disseminação da tuberculose intra e extramuros é a relação estabelecida pela PPL com os profissionais do local, visitantes, entre os presos e, quando já libertados, a relação com a comunidade. Em sua maioria esses indivíduos são oriundos de áreas com infraestrutura precária e dificuldade de acesso aos serviços (OLIVEIRA et al., 2015b).

Existem várias abordagens para estudar as iniquidades em saúde provocadas pelos DSS, podemos verificar que a que privilegia os aspectos físico-materiais, no que se refere à ausência de investimentos em infraestrutura comunitária, educação, transporte, saneamento, habitação, serviços de saúde entre outros, e são resultantes de processo econômico e de decisão política (BUSS; PELLEGRINI FILHO, 2007), é a que melhor se aplica neste estudo. De maneira geral, observamos que não há como não falar em iniquidades em saúde quando parte da PPL é oriunda de áreas sem infraestrutura e inserida em um ambiente onde estes serviços e condições também são precários.

Os estudos de Navarro et al. (2016) e Oliveira et al. (2015a) indicam que a PPL, em sua maioria, é composta por jovens adultos, com menos escolaridade, de comunidades menos favorecidas e com taxas altas de tuberculose ativa.

Já em Oliveira et al. (2015b), notamos que na pesquisa foram analisadas, também, as condições de vida em relação à vulnerabilidade sociodemográfica à infecção e adoecimento por tuberculose, em que, no caso 1, percebeu-se alta den- 
sidade demográfica e proporção de residentes em aglomerados subnormais semelhante à taxa nacional e, no caso 2, percebeu-se alta densidade tanto demográfica quanto em proporção de residentes em aglomerados subnormais, que são favelas, invasões, comunidades, vilas entre outros assentamentos irregulares.

As condições de saúde do indivíduo são pautadas pelo impacto dos DSS intermediários, compostos por condições de vida, fatores comportamentais, biológicos, psicossociais e o sistema de saúde. Segundo documento técnico publicado pela OMS, em 2011, os mecanismos que alteram a posição social dos indivíduos são a mais profunda causa das iniquidades em saúde, e estes, por sua vez, estão relacionados às desigualdades injustas e evitáveis (OMS, 2011).

Cordeiro et al. (2018) sinalizam que é importante frisar que a PPL não recebe orientações acerca das doenças infectocontagiosas, levando assim a conceitos distorcidos quanto a transmissão, sintomas, tratamento e cura, principalmente em relação à tuberculose. Todavia, com orientação e controle, a prevalência e o abandono ao tratamento devem diminuir.

\section{Considerações finais}

A tuberculose continua sendo uma das principais causas de mortalidade por doença infectocontagiosa no mundo (MAFFACCIOLLI et al., 2015) e seu diagnóstico e tratamento não podem desconsiderar o cenário de vulnerabilidade em saúde resultante da articulação entre desigualdades sociais, aspectos biológicos e manifestações comportamentais percebidos na população infectada (MAFFACCIOLLI et al., 2017).

O estudo evidenciou que o Estado, enquanto tutor das pessoas que se encontram em cumprimento de penas em privação de liberdade, não assegura as condições de dignidade da pessoa humana em seu acesso à saúde. Esta afirmação se dá com base na não garantia percebida em relação aos direitos assegurados pela LEP, nos inúmeros relatos quanto às questões de insalubridade e más condições das celas, má ventilação e superlotação.

Apesar da indicação do PNSSP, o TDO não é realizado na maioria dos estudos, o que, como já apontado, pode inviabilizar o tratamento e cura desses pacientes, quando aliamos este fato com a escassez de medicamentos e de materiais e insumos, a garantia do tratamento fica ainda mais comprometida.

Não podemos deixar de citar que o cadastramento da PPL junto aos estabelecimentos de saúde é de extrema importância, tanto para o controle em relação ao agravo, quanto para que ocorra o fornecimento correto desses materiais e medicamentos, que já são também prejudicados devido ao alto fluxo de ingresso dessa população.

O quadro defasado dos profissionais de saúde, assim como a falta de treinamento contínuo e adequado desses profissionais, aliado às condições de segurança e dependência de um olhar mais cuidadoso dos agentes de segurança em relação à saúde da PPL, dificultam que se faça uma correta orientação mínima a essa população que, conforme estudos, já vem de áreas menos favorecidas, com menos condição de acesso e esclarecimentos acerca dos processos de saúde e doença.

Dessa forma, faz-se necessário que sejam realizadas capacitações aos profissionais de saúde, para alinhamento aos programas e planos implementados para a saúde da PPL, para que se tenha maior segurança destes em relação aos procedimentos que devem ser realizados, bem como, para que possam expor as dificul- 
dades encontradas na rotina de atendimento. Necessita também que sejam incluídos os agentes de segurança, para que estes possam compreender o seu papel nos serviços de saúde e o quanto essas medidas impactam não apenas a vida e a saúde da PPL, como também a de todos os profissionais que laboram nas unidades penitenciárias, familiares (intra e extramuros), advogados e toda a população.

Não podemos nos furtar de citar, que metade dos estudos sinaliza que a maioria da população analisada provém de um contexto em que as iniquidades em saúde se fazem presentes cotidianamente, mostrando a necessidade de ações sobre os determinantes dessas iniquidades. Não há como pensar em políticas de saúde para a PPL sem considerar as origens dessas populações, e como esses fatores influenciam o sistema de encarceramento no Brasil e a relação dos DSS no quadro de saúde e doença dessa população.

A insuficiência de publicações científicas em saúde pública que articulem as noções de risco e vulnerabilidade à temática da tuberculose, levando em consideração os elementos psicossociais envoltos no processo de adoecimento, diagnóstico e tratamento mostrou-se um entrave no desenvolvimento do presente estudo. O quadro é ainda mais insatisfatório quando se busca dados que conectem a tuberculose ao sistema penitenciário no estado de Mato Grosso. Exatamente por isso, esta pesquisa mostra-se relevante e necessária e, apesar das dificuldades envolvidas em sua realização, conseguiu reunir os principais pontos deficitários no acesso e garantia dos direitos a saúde da população privada de liberdade no Brasil.

Importante ressaltar a necessidade de mais estudos sobre a tuberculose no sistema penitenciário brasileiro e estadual que analisem os DSS da PPL, para que seja possível aliar os planos e programas já existentes ao processo de identificação das dificuldades de implantação e controle desses, possibilitando, assim, que seja resguardado o direito dessas pessoas em seu acesso à saúde.

Recebido em 10 de dezembro de 2020.

Aceito em 15 de março de 2021.

\section{Referências}

ADORNO, Sérgio. O sistema penitenciário no Brasil: problemas e desafios. $R e-$ vista USP, 9: 65-78, 1991.

ANDRADE, U. S.; FERREIRA, F. F. Crise no sistema penitenciário brasileiro: capitalismo, desigualdade social e prisão. Revista de Psicologia, Diversidade e Saúde, 3 (1): 116-129, 2014.

ASSIS, Rafael Damaceno. A realidade atual do sistema penitenciário brasileiro. Revista CEJ, 39: 74-78, 2007. 
BARBOSA, M. L.; CELINO, S. D. M.; VERÍSSIMO E OLIVEIRA, L.; PEDRAZA, D. F.; COSTA, G. M. C. Atenção básica à saúde de apenados no sistema penitenciário: subsídios para a atuação da enfermagem. Escola Anna Nery, 18 (4): 586592, 2014.

BRASIL. Ministério da Justiça. 1984. Lei n⿳0 7.210, de 11 de julho de 1984. Institui a Lei de Execução Penal. Disponível em: <http://www.planalto.gov.br/ccivil_03/LEIS/L7210.htm>. Acesso em: 15 jul. 2019.

BRASIL. Biblioteca Virtual em Saúde do Ministério da Saúde. $8^{\text {a }}$ Conferência Nacional de Saúde. Brasília: MS, 1986.

BRASIL. Ministério da Saúde. Secretaria de Atenção à Saúde. Departamento de Ações Programáticas Estratégicas. Área Técnica de Saúde no Sistema Penitenciário. Plano Nacional de Saúde no Sistema Penitenciário. Brasília: Ministério da Saúde, 2004.

BRASIL. Ministério da Saúde. Secretaria de Vigilância em Saúde. Departamento de Vigilância Epidemiológica. Doenças Infecciosas e Parasitárias: guia de bolso. Brasília: Ministério da Saúde, 2010.

BRASIL. Conselho Nacional de Saúde. Resolução no 466, de 12 de dezembro de 2012. Diretrizes e normas regulamentadoras para pesquisas envolvendo seres humanos (revoga as seguintes resoluções: 196/96, 404/o8 e 303/oo). Brasília: CNS, 2012.

BRASIL. Ministério da Saúde. 2014. Lei $\mathrm{n}^{0}$ 1, de 2 de janeiro de 2014. Institui a Política Nacional de Ação Integral à Saúde das Pessoas Privadas de Liberdade no Sistema Prisional (PNAISP).

BRASIL. Ministério da Saúde. Secretaria de Vigilância em Saúde. Departamento de Vigilância das Doenças Transmissíveis. Brasil Livre da Tuberculose: Plano Nacional pelo Fim da Tuberculose como Problema de Saúde Pública. Brasília: Ministério da Saúde, 2017.

BRASIL. Ministério da Saúde. Secretaria de Vigilância em Saúde. Departamento de Vigilância das Doenças Transmissíveis. Manual de Recomendações para o Controle da Tuberculose no Brasil. Brasília: Ministério da Saúde, 2018.

BRASIL. Ministério da Saúde. Secretaria de Vigilância em Saúde. Departamento de Vigilância Epidemiológica. Programa Nacional de Controle da Tuberculose.

BUSS, P. M.; PELLEGRINI FILHO, A. A saúde e seus determinantes sociais. Physis: Revista de Saúde Coletiva, 17 (1): 77-93, 2007.

ARDINS, K. K. B.; FREITAS, C. H. S. M.; SIMÕES, M. O. S.; COSTA, G. M. C. Acesso e uso racional de medicamentos no sistema prisional da Paraíba. Escola Anna Nery, 23 (2): 1-9, 2019.

CORDEIRO, E. L.; SILVA, T. M.; SILVA, L. S. R.; PEREIRA, C. E. A.; PATRICIO, F. B.; SILVA, C. M. Perfil epidemiológico dos detentos: patologias notificáveis. Avances em Enfermagem, 36 (2): 170-178, 2018.

DIUANA, V.; LHUILIER, D.; SÁNCHEZ, A. R.; AMADO, G.; ARAÚJO, L.; DUARTE, A. M.; GARCIA, M.; MILANEZ, E.; POUBEL, L.; ROMANO, E.; LAROUZÉ, B. Saúde em prisões: representações e práticas dos agentes de segurança penitenciária no Rio de Janeiro, Brasil. Cadernos de Saúde Pública, 24 (8): 18871896, 2008. 
FOUCALT, Michel. Vigiar e punir: nascimento da prisão. Petrópolis: Vozes, 1977.

FOUCALT, Michel. Microfísica do poder. Rio de Janeiro: Edições Graal, 1979.

GOFFMAN, Erving. Manicômios, prisões e conventos. São Paulo: Perspectiva, 1961. MAFFACCIOLLI, R.; HAHN, G. V.; ROSSETTO, M.; ALMEIDA, C. P. B.; MANICA, S. T.; PAIVA, T. S.; OLIVEIRA, D. L. L. C. A utilização da noção de vulnerabilidade na produção de conhecimento sobre tuberculose: revisão integrativa. Revista Gaúcha de Enfermagem, 36 (n. spe): 247-253, 2015.

MAFFACCIOLLI, R.; OLIVEIRA, D. L.; BRAND, E. M. Vulnerabilidade e direitos humanos na compreensão de trajetórias de internação por tuberculose. Saúde e Sociedade, 26 (1): 286-299, 2017.

MENDES, K. D. S.; SILVEIRA, R. C. C. P.; GALVÃO, C. M. Revisão integrativa: método de pesquisa para a incorporação de evidências na saúde e na enfermagem. Texto \& Contexto - Enfermagem, 17 (4): 758-764, 2008.

MINISTÉRIO DA SAÚDE. Tuberculose: o que é, causas, sintomas, tratamento, diagnóstico e prevenção. Disponível em: <http://www.saude.gov.br/saude-de-az/tuberculose\#populacao $>$. Acesso em: jul. 2019.

MINISTÉRIO DA SAÚDE. Política Nacional de Atenção Integral à Saúde das Pessoas Privadas de Liberdade no Sistema Prisional (PNAISP). Disponível em: $<$ http://www.saude.gov.br/acoes-e-programas/pnaisp/politica-nacional-deatencao-integral-a-saude-das-pessoas-privadas-de-liberdade-no-sistema-prisional>. Acesso em: jul. 2019.

NAVARRO, P. D; ALMEIDA, I. N.; KRITSKI, A. L.; CECCATO, M. G.; MACIEL, M. M. D.; CARVALHO, W. S.; MIRANDA, S. S. Prevalência da infecção latente por Mycobacterium tuberculosis em pessoas privadas de liberdade. Jornal Brasileiro de Pneumologia, 42 (5): 348-355, 2016.

OLIVEIRA, L. G. D.; NATAL, S.; CAMACHO, L. A. B. Análise da implantação do Programa de Controle da Tuberculose em unidades prisionais no Brasil. Cadernos de Saúde Pública, 31 (3): 543-554, 2015 a.

OLIVEIRA, L. G. D.; NATAL, S.; CAMACHO, L. A. B. Contextos de implantação do Programa de Controle da Tuberculose nas prisões brasileiras. Revista de Saúde Pública, 49 (66): 1-9, 2015b.

OMS - Organização Mundial de Saúde. Diminuindo diferenças: a prática das políticas sobre determinantes sociais da saúde: documento de discussão. Rio de Janeiro, Brasil, out. de 2011. Disponível em: <http://cmdss2011.org/site/wp-content/uploads/2011/10/Documento-Tecnico-da-Conferencia-vers\%C3\%A3o-final.pdf $>$. Acesso em: 03 mar. 2020.

SÁNCHEZ, A. R.; CAMACHO, L. A. B.; DIUANA, V.; LAROUZÉ, B. A tuberculose nas prisões: uma fatalidade? Cadernos de Saúde Pública, 22 (12): 2510-2511, 2006.

SOARES, C. B.; HOGA, L. A. K.; PEDUZZI, M.; SANGALETI, C.; YONEKURA, T.; SILVA, D. R. A. D. Revisão integrativa: conceitos e métodos utilizados na enfermagem. Revista da Escola de Enfermagem da USP, 48 (2): 335-345, 2014.

SOARES FILHO, M. M.; BUENO, P. M. M. G. Demografia, vulnerabilidades e direito à saúde da população prisional brasileira. Ciência \& Saúde Coletiva, 21 (7): 1999-2010, 2016. 
SOUZA, M. T.; SILVA, M. D.; CARVALHO, R. Revisão integrativa: o que é e como fazer. Einstein, 8 (1): 102-106, 2010.

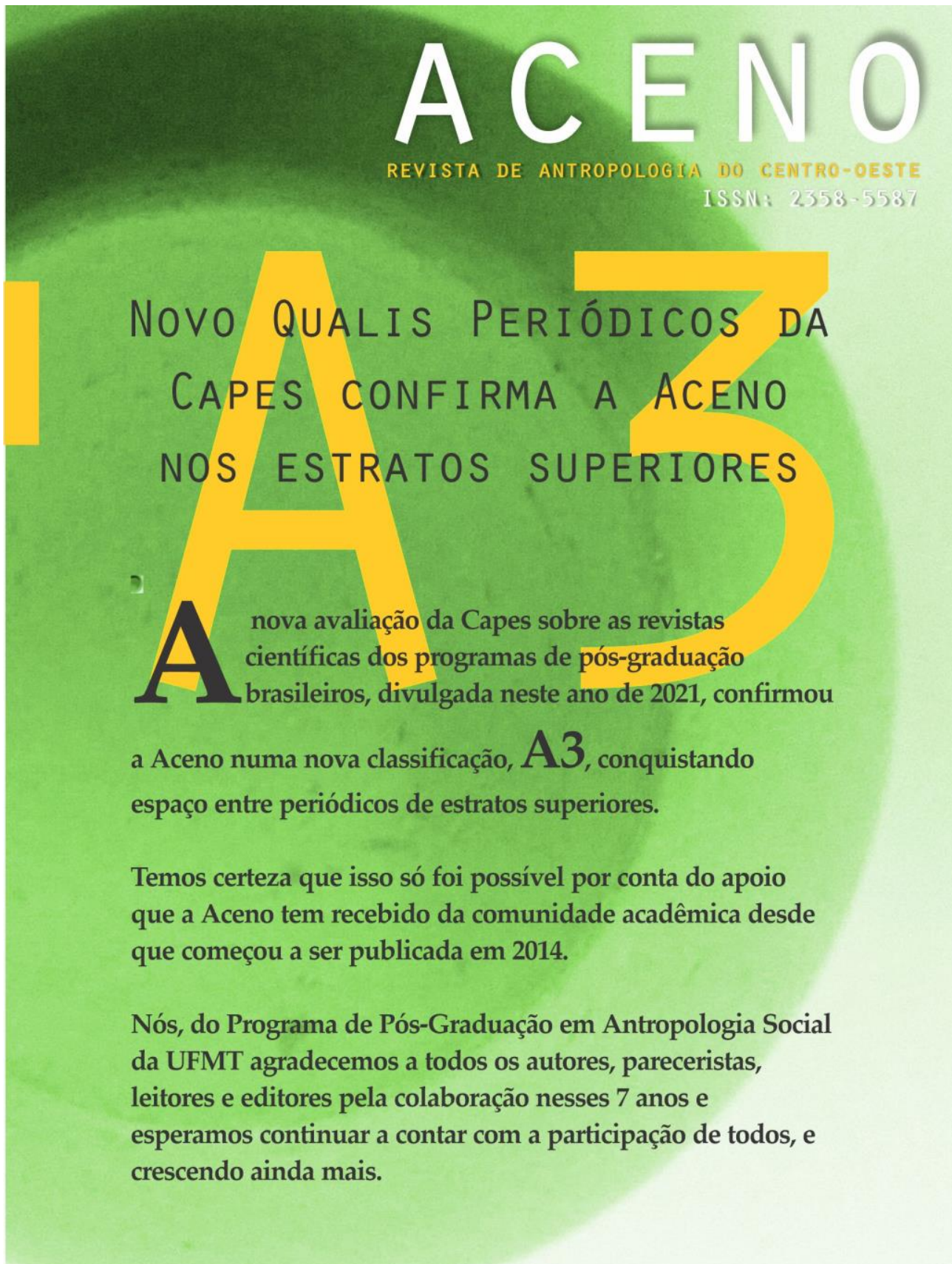

